\title{
Small nucleolar RNAs are concomitantly down-regulated during murine gamma delta T- cell development.
}

\author{
Shahan Mamoor ${ }^{1}$ \\ 1Thomas Jefferson School of Law \\ San Diego, CA 92101 \\ mamoorsk@tjsl.edu
}

Gamma delta T-cells are a lymphocyte subset that display gamma delta T-cell receptors rather than the alpha beta T-cell receptors that alpha beta T cells like CD4 helper and CD8 cytotoxic T-cells display, and whose function straddles the intersection of innate and adaptive immune cells (1). To understand the transcriptional behavior of gamma delta T-cells during mammalian development, we performed global comparative differential gene expression profiling of datasets encompassing transcriptome data from embryonic and adult gamma delta T-cells from mice (2). These analyses revealed a species of non-coding RNA termed small nucleolar RNA, or snoRNA were among the most differentially expressed genes when comparing embryonic and adult gamma delta T-cells. Moreover, these snoRNA were uniformly downregulated over the course of gamma delta T-cell development. These data demonstrate unprecedented developmental repression of snoRNA in lymphocytes and suggest that stagespecific repression of snoRNAs may serve some vital developmental purpose in the function of gamma delta T-cells.

Keywords: gamma delta T-cells, snoRNA, non-coding RNA, lymphocyte development, $\gamma \delta$ T-

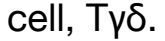




\section{Introduction}

Gamma delta T-cells are a type of T-lymphocyte that are defined by their usage of gamma and delta chains of the Tcr gene locus at the T-cell receptor $(3,4)$. They inhabit diverse anatomical sites such as the skin, the blood, thymus, tonsils and the spleen (5), and perform diverse functions from specific recognition of organic ligands (6) to parasite defense (7). The developmental origin of gamma delta T-cells is considered identical to that of alpha beta T-cells until sometime during the double negative 2 (DN2) or DN3 thymocyte maturation stages wherein beta selection, the process by which thymocytes express the beta chain of the TCR and choose the alpha beta lineage, at which point the lineage of the alpha beta T-cell diverges from that of the gamma delta T-cell $(8,9,10,11,12,13,14)$.

To define the most significant transcriptional changes in the developmental program of gamma delta T-cells, we performed comparative gene expression profiling of mammalian gamma delta T-cells from embryonic day 18 and 6 weeks of age from mice using a published dataset (2). This analysis revealed that a class of non-coding RNA or ncRNA termed small nucleolar RNAs, or snoRNAs are differentially expressed in a stage-specific fashion and that these snoRNA transcripts uniformly decrease in expression through development.

\section{Methods}

GSE71637 (2) was utilized for this differential gene expression analysis, performed using the GEO2R web application, which identifies the most differentially expressed genes in any given comparison. Muñoz-Ruiz et al. used Affymetrix Mouse Gene 2.1 ST Array to perform global transcriptional profiling in wild-type and $\mathrm{CD} 3 \mathrm{H}$ deficient mice; these data were extracted from this dataset. For all analyses on GEO2R, p-value adjustment was set to "Benjamini and Hochberg", log-transformation of data was set to "auto-detect", and the "NCBI generated" category of platform annotation was selected. Statistical analysis was performed with PRISM 8.1.2 (227) using an unpaired t-test with Welch's correction. 


\section{Results}

snoRNA are among the most differentially expressed genes when comparing gamma delta T-cells across development.

We used GEO2R to perform comparative global differential gene expression analysis, to identify the genes whose expression is most different between day 18 embryonic gamma delta T-cells and adult gamma delta T-cells at 6 weeks of age. Twenty-seven of the top five hundred most differentially expressed genes were ncRNA species including anti-sense RNA transcripts, pseudogenes, small nuclear RNA (snRNA), and microRNA (miRNA) (2) (S. Mamoor, analysis unpublished). Small nucleolar RNA, or snoRNA represented twelve of these ncRNA (Table 1). These snoRNA included snord16a, snord82, and snord87 as well as nine other snoRNA transcripts (Table 1 and Figure 2).

\section{Of the most differentially expressed non-coding RNA, snoRNA but not miRNA are} uniformly down-regulated during gamma delta T-cell development.

Analysis of the most differentially expressed ncRNA transcripts across murine gamma delta T-cell development revealed that while snoRNA and miRNA transcripts revealed that while the expression of these differentially expressed miRNA both increased and decreased through development (Figure 1B and Figure 3), the expression of each of the twelve most differentially expressed snoRNAs strictly decreased over development (Figure 1A and Figure 2).

\section{Discussion}

The central dogma of molecular biology (15) is that genes encoded by DNA are transcribed by transcriptional machinery (e.g., RNA polymerases) in a process known as transcription, and the resultant RNA templates are, in a process known as translation, translated into protein by machinery including the signal recognition particle, smooth and rough ribosomes and tRNA. It has since become apparent that some RNAs do not translate into 
proteins; these transcripts are known as non-coding RNA (16). Though the function of many non-coding RNA remains elusive, the function of some has been elucidated. Xist is a noncoding RNA that functions in the maternal imprinting process (17), wherein one copy of the $\mathrm{X}$ chromosome is silenced by coating of that chromosome with Xist non-coding RNA transcripts, which recruits further epigenetic machinery like the polycomb repressive complex PRC2 $(18,19)$, which methylates lysine 27 of Histone H3 (H3K27) on chromosomal DNA in order to enforce heterochromatin formation (compaction into dense, inactive DNA). Another noncoding RNA, Hotair similarly recruits PRC2, as well as the H3K4 lysine specific demethylase LSD1 to the HoxD locus $(20,21)$, and becomes significantly active in cancers of the pancreas, breast, and stomach $(22,23,24,25)$.

The nucleolus is a sub-organelle within the nucleus where specific transcription of ribosomal RNA, including $18 \mathrm{~S}$ and $28 \mathrm{~S}$ rRNA occurs (26). The nucleolus has specific targeting signals associated with transport and delivery of molecules therein, including PLLKKIKQ for cmyb, PQPKKKP for p53, GRKKRRQRRRAP for the HIV-1 Tat protein (27) and RRRGL for angiogenin (28). SnoRNA are a class of non-coding RNA that are associated with the nucleolus (29).

SnoRNAs can be generally be classified into two main groups, known as C/D box and H/ACA box snoRNAs (29). snoRNAs have been described to facilitate site-specific pseudouridylation and 2'-O-methylation of rRNAs (30). C/D box snoRNAs function as guiding templates for 2'-O-methylation of rRNA, while H/ACA box snoRNAs function likewise for the psuedouridylation of rRNAs $(31,32)$. Though one study has identified a species of non-coding RNA known as sno-derived RNA, or sdRNA as markers of cancer immunity (33), there is no known role for snoRNA in the function or development of gamma delta T-cells, nor has it been reported that snoRNA are differentially expressed in gamma delta T-cells. In mouse macrophages, a long intergenic non-coding RNA lincRNA-Cox2 has been described to function in the activation and repression of immune genes in vitro and in vivo $(34,35)$, but a 
similar role or function has not yet been defined for snoRNA in lymphocytes, in general, nor could we identify any literature describing a role for any type of ncRNA in gamma delta T-cells.

These results demonstrate not only that snoRNA are among the most differentially expressed genes in murine gamma delta T-cell development, but that the expression of these differentially expressed snoRNA uniformly decreases over time; in this study, from embryonic day 18 to 6 weeks of age. While miRNA were also differentially expressed (seven in total), the expression of these differentially expressed miRNA both increased and decreased over gamma delta T-cell development. Twelve separate snoRNA were identified in this global differential gene expression analysis of murine gamma delta T-cells. The function of these snoRNA in gamma delta T-cells is unknown. It is possible that site-specific pseudouridylation or 2'-Omethylation of rRNA or some other substrate RNA message may be essential for gamma delta T-cell development, or that repression of site-specific pseudouridylation or 2'-O-methylation of rRNA or some other substrate RNA message may be required for the developmental transition from embryonic to adult gamma T-cell.

To our knowledge, these are the first data to demonstrate differential and stageselective expression of snoRNA during gamma delta T-cell development. These results merit further investigation into the role of snoRNA during lymphocyte development, specifically of the gamma delta T-cell. 


\section{References}

1. Born, Willi K., Christopher L. Reardon, and Rebecca L. O’Brien. "The function of $\gamma \delta T$ cells in innate immunity." Current opinion in immunology 18.1 (2006): 31-38.

2. Muñoz-Ruiz, Miguel, et al. "TCR signal strength controls thymic differentiation of discrete proinflammatory $\gamma \delta ~ T$ cell subsets." Nature immunology 17.6 (2016): 721.

3. Ishida, Isao, et al. "T-cell receptor gamma delta and gamma transgenic mice suggest a role of a gamma gene silencer in the generation of alpha beta T cells." Proceedings of the National Academy of Sciences 87.8 (1990): 3067-3071.

4. Haas, Werner, Pablo Pereira, and Susumu Tonegawa. "Gamma/delta cells." Annual review of immunology 11.1 (1993): 637-685.

5. Falini, B., et al. "Distribution of T cells bearing different forms of the T cell receptor gamma/ delta in normal and pathological human tissues." The Journal of Immunology 143.8 (1989): 2480-2488.

6. Belmant, Christian, et al. "A chemical basis for selective recognition of nonpeptide antigens by human $\delta$ T cells." The FASEB Journal 14.12 (2000): 1669-1670.

7. Elloso, M. Merle, et al. "Inhibition of Plasmodium falciparum in vitro by human gamma delta T cells." The Journal of Immunology 153.3 (1994): 1187-1194.

8. Burtrum, Douglas B., et al. "TCR gene recombination and alpha beta-gamma delta lineage divergence: productive TCR-beta rearrangement is neither exclusive nor preclusive of gamma delta cell development." The Journal of Immunology 157.10 (1996): 4293-4296.

9. Ciofani, Maria, et al. "Stage-specific and differential notch dependency at the $a \beta$ and $\gamma \delta T$ lineage bifurcation." Immunity 25.1 (2006): 105-116.

10. Aifantis, lannis, et al. "On the Role of the Pre-T Cell Receptor in $a \beta$ versus $\gamma \delta ~ T$ Lineage Commitment." Immunity 9.5 (1998): 649-655.

11. Tanigaki, Kenji, et al. "Regulation of $a \beta / \gamma \delta T$ cell lineage commitment and peripheral $T$ cell responses by Notch/RBP-J signaling." Immunity 20.5 (2004): 611-622.

12. Kreslavsky, Taras, et al. "T cell receptor-instructed $a \beta$ versus $\gamma \delta$ lineage commitment revealed by single-cell analysis." Journal of Experimental Medicine 205.5 (2008): 1173-1186.

13. Garbe, Annette I., et al. "Differential synergy of Notch and T cell receptor signaling determines a $\beta$ versus $\gamma \delta$ lineage fate." Journal of Experimental Medicine 203.6 (2006): 1579-1590.

14. Ciofani, Maria, and Juan Carlos Zúñiga-Pflücker. "Determining $\gamma \delta$ versus a $\beta$ T cell development." Nature Reviews Immunology 10.9 (2010): 657.

15. Crick, Francis. "Central dogma of molecular biology." Nature 227.5258 (1970): 561.

16. Mattick, John S., and Igor V. Makunin. "Non-coding RNA." Human molecular genetics 15.suppl_1 (2006): R17-R29.

17. Penny, Graeme D., et al. "Requirement for Xist in X chromosome inactivation." Nature 379.6561 (1996): 131.

18. Sarma, Kavitha, et al. "ATRX directs binding of PRC2 to Xist RNA and Polycomb targets." Cell 159.4 (2014): 869-883. 
19. da Rocha, Simão Teixeira, et al. "Jarid2 is implicated in the initial Xist-induced targeting of PRC2 to the inactive X chromosome." Molecular cell 53.2 (2014): 301-316.

20. Wu, Liang, et al. "Binding interactions between long noncoding RNA HOTAIR and PRC2 proteins." Biochemistry 52.52 (2013): 9519-9527.

21. Li, Lingjie, et al. "Targeted disruption of Hotair leads to homeotic transformation and gene derepression." Cell reports 5.1 (2013): 3-12.

22. Kim, Kyounghyun, et al. "HOTAIR is a negative prognostic factor and exhibits prooncogenic activity in pancreatic cancer." Oncogene 32.13 (2013): 1616.

23. Gupta, Rajnish A., et al. "Long non-coding RNA HOTAIR reprograms chromatin state to promote cancer metastasis." Nature 464.7291 (2010): 1071.

24. Sørensen, Kristina P., et al. "Long non-coding RNA HOTAIR is an independent prognostic marker of metastasis in estrogen receptor-positive primary breast cancer." Breast cancer research and treatment 142.3 (2013): 529-536.

25. Liu, Xiang-hua, et al. "Lnc RNA HOTAIR functions as a competing endogenous RNA to regulate HER2 expression by sponging miR-331-3p in gastric cancer." Molecular cancer 13.1 (2014): 92.

26. Boisvert, François-Michel, et al. "The multifunctional nucleolus." Nature reviews Molecular cell biology 8.7 (2007): 574.

27. Dang, Ch V., and W. M. Lee. "Nuclear and nucleolar targeting sequences of c-erb-A, cmyb, N-myc, p53, HSP70, and HIV tat proteins." Journal of Biological Chemistry 264.30 (1989): 18019-18023.

28. Moroianu, Junona, and James F. Riordan. "Identification of the nucleolar targeting signal of human angiogenin." Biochemical and biophysical research communications 203.3 (1994): 1765-1772.

29. Samarsky, Dmitry A., et al. "The snoRNA box C/D motif directs nucleolar targeting and also couples snoRNA synthesis and localization." The EMBO Journal 17.13 (1998): 3747-3757.

30. Kiss, Tamás. "Small nucleolar RNA-guided post-transcriptional modification of cellular RNAs." The EMBO journal 20.14 (2001): 3617-3622.

31. Ni, Jingwei, Amy L. Tien, and Maurille J. Fournier. "Small nucleolar RNAs direct sitespecific synthesis of pseudouridine in ribosomal RNA." Cell 89.4 (1997): 565-573.

32. Kiss-László, Zsuzsanna, et al. "Site-specific ribose methylation of preribosomal RNA: a novel function for small nucleolar RNAs." Cell 85.7 (1996): 1077-1088.

33. Chow, Ryan D., and Sidi Chen. "Sno-derived RNAs are prevalent molecular markers of cancer immunity." Oncogene 37.50 (2018): 6442.

34. Atianand, Maninjay K., and Katherine A. Fitzgerald. "Long non-coding RNAs and control of gene expression in the immune system." Trends in molecular medicine 20.11 (2014): 623-631.

35. Carpenter, Susan, et al. "A long noncoding RNA mediates both activation and repression of immune response genes." science 341.6147 (2013): 789-792. 


\section{Table 1}

\begin{tabular}{|l|r|r|l|l|}
\hline Species & \multicolumn{1}{l}{ Rank } & \multicolumn{1}{l|}{ p-value } & Chromosome & ID \\
\hline ENSMUST00000157287 // ncRNA:snoRNA & 20 & 0.00004042 & chr14 & 17304512 \\
\hline ENSMUST00000104252 // ncRNA:snoRNA & 43 & 0.00006299 & chr10 & 17239755 \\
\hline ENSMUST00000157820 // ncRNA snoRNA & 53 & 0.00007607 & chrX & 17543194 \\
\hline small nucleolar RNA C/D box 87 Snord87 & 91 & 0.00011523 & chr1 & 17221191 \\
\hline ENSMUST00000083425 // ncRNA:snoRNA & 118 & 0.00014807 & chr9 & 17518316 \\
\hline ENSMUST00000158284 //ncRNA:snoRNA & 126 & 0.0001615 & chr19 & 17356425 \\
\hline Snord16a & 129 & 0.00016562 & chr9 & 17518312 \\
\hline ENSMUST00000082608 // ncRNA:snoRNA & 155 & 0.00019557 & chr4 & 17428795 \\
\hline ENSMUST00000104449 // ncRNA:snoRNA & 157 & 0.00019969 & chr11 & 17252847 \\
\hline ENSMUST00000082738 // ncRNA:snoRNA & 228 & 0.00029172 & chr13 & 17287336 \\
\hline ENSMUST00000116972 // ncRNA:snoRNA & 265 & 0.00036898 & chr2 & 17374316 \\
\hline Snord82 & 289 & 0.00041772 & chr1 & 17225173 \\
\hline
\end{tabular}

Table 1: snoRNA are among the most differentially expressed genes when comparing murine gamma delta T-cells across development.

The rank of each snoRNA transcript based on statistical significance of global differential expression, the corresponding $\mathrm{p}$-value, chromosomal location of the gene encoding that transcript, and transcript ID provided by NCBI GEO2R are shown. 


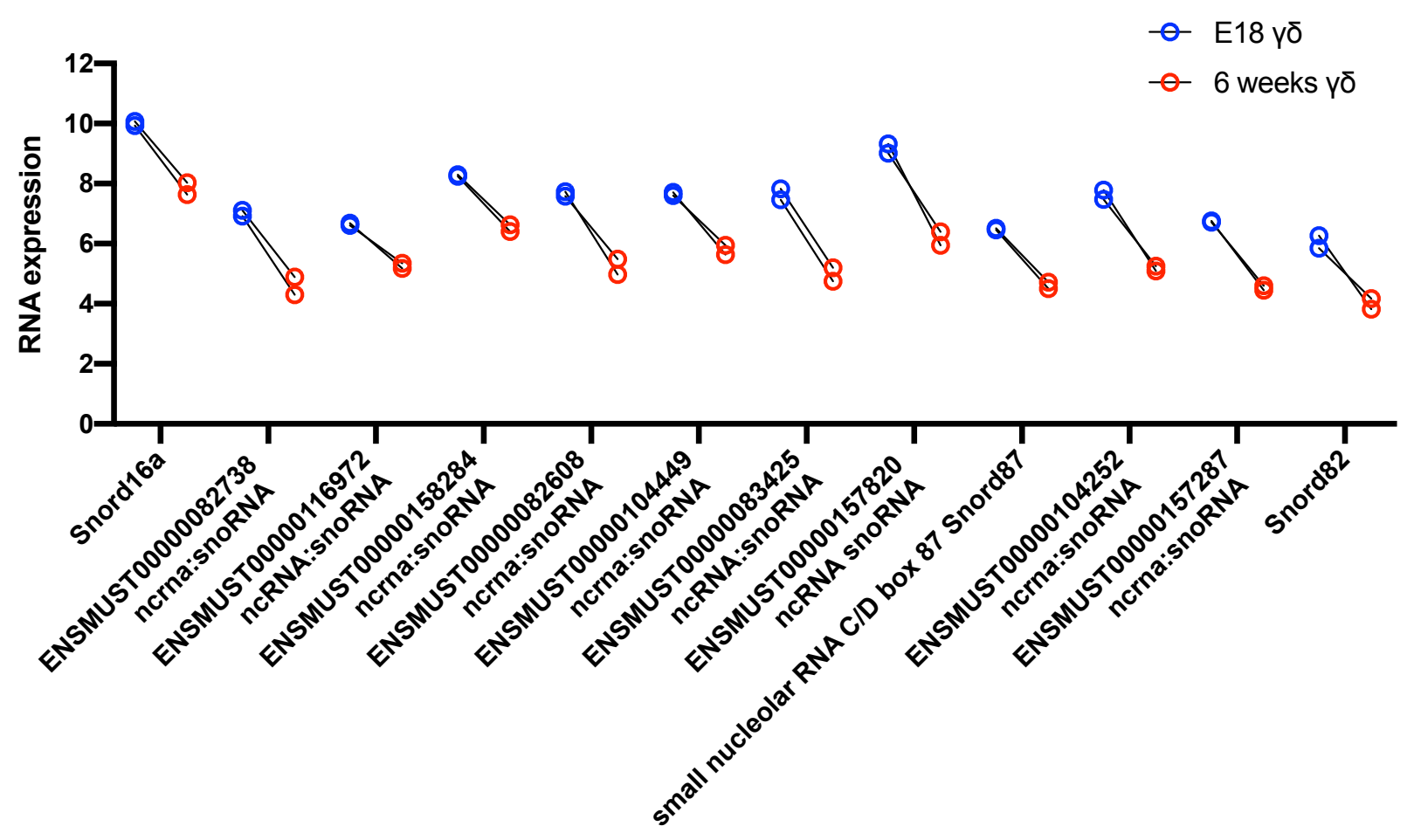

Figure 1: snoRNA but not miRNA are differentially expressed and down-regulated in a developmental stage-specific manner in murine gamma delta T-cells.

Figure 1A: The RNA expression value of each differentially expressed snoRNA transcript, in wild-type and CD3H deficient mice ( $n=2$ for developmental stage; each $n$ of 2 includes 1 sample each from day 18 embryos and adult mice 6 weeks of age) is displayed. 


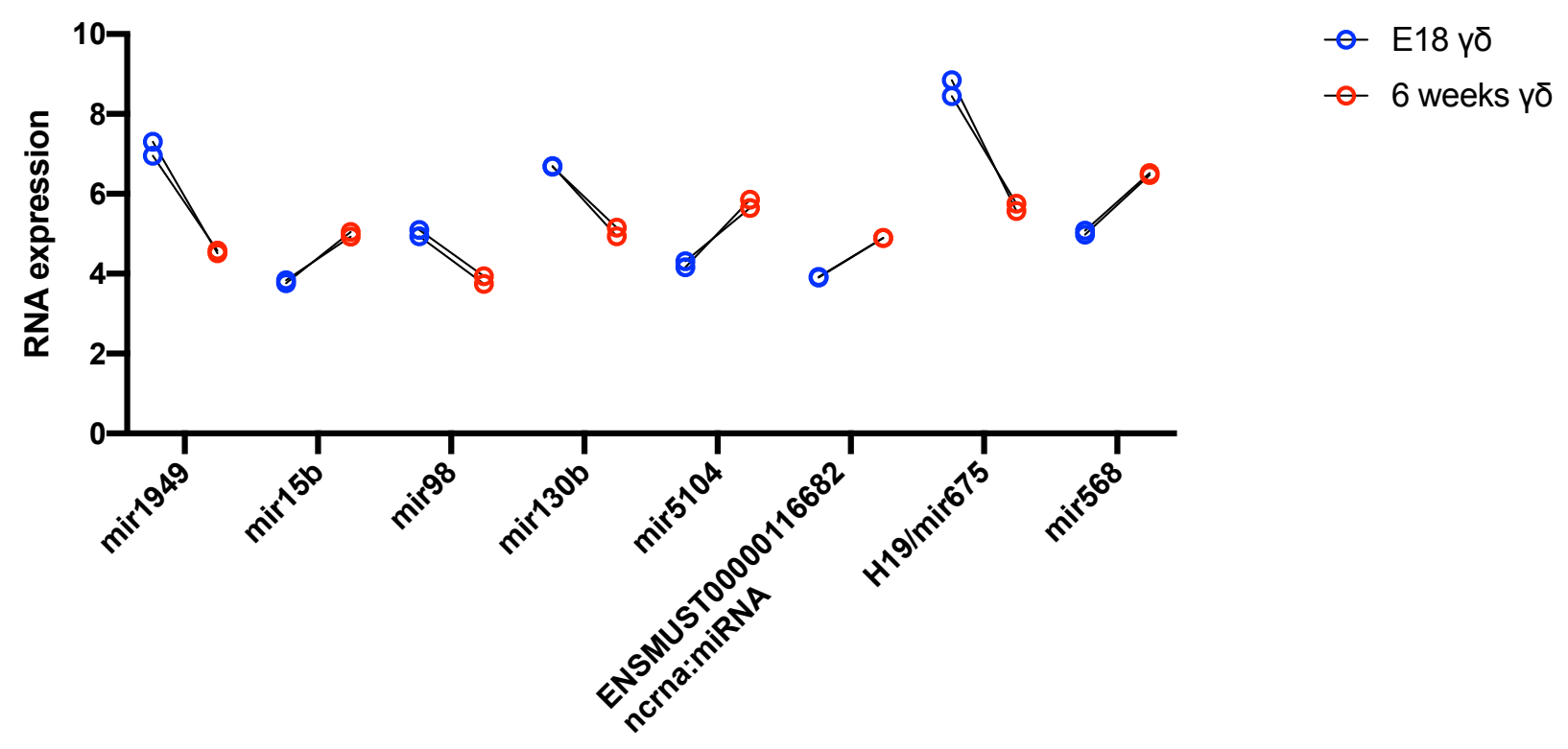

Figure 1: snoRNA but not miRNA are differentially expressed and down-regulated in a developmental stage-specific manner.

Figure 1B: The RNA expression value of each differentially expressed miRNA transcript, in wild-type and CD3H deficient mice ( $n=2$ for developmental stage; each $n$ of 2 includes 1 sample from day 18 embryos and adult mice 6 weeks of age) is displayed. 

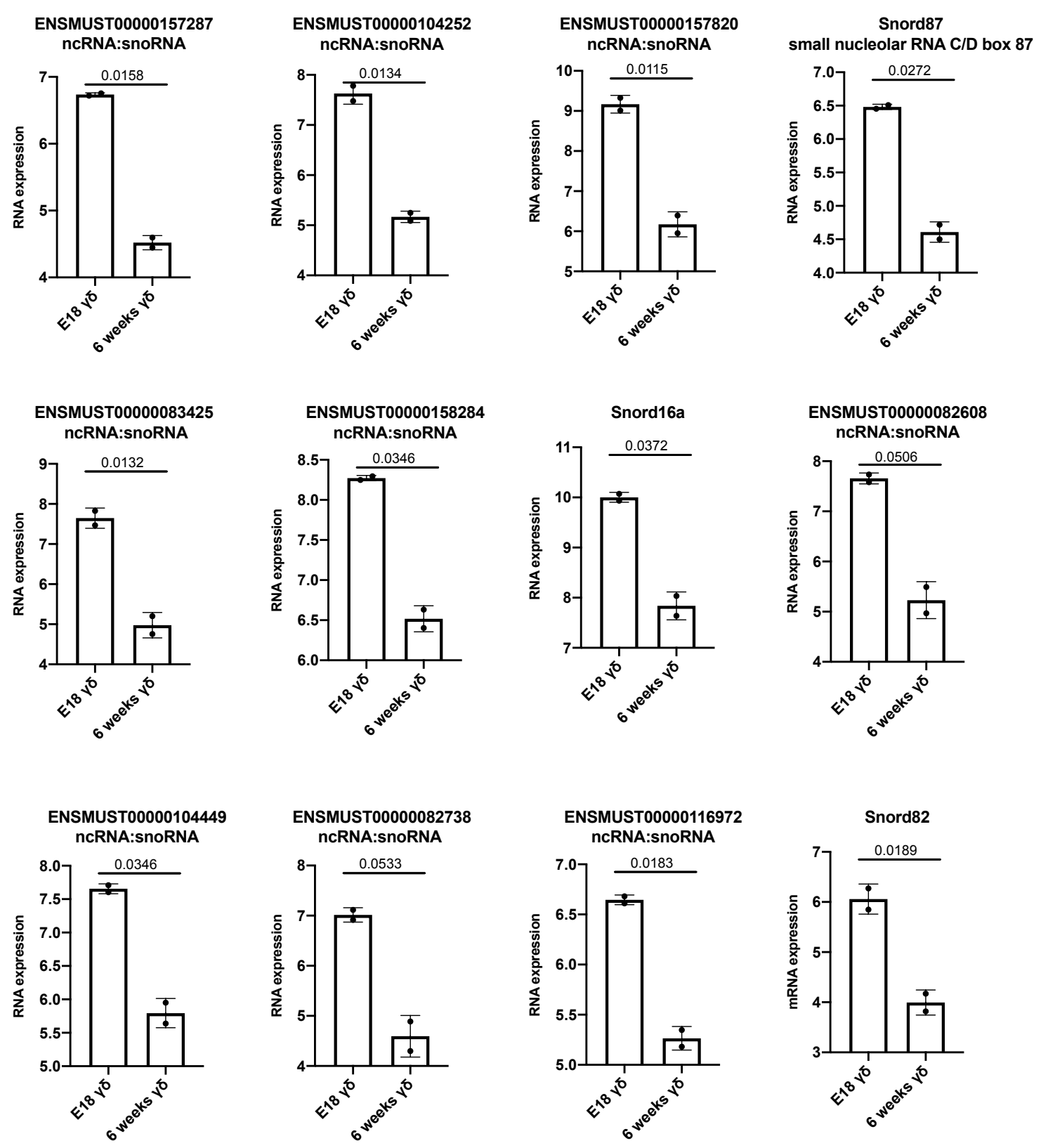

Figure 2: snoRNA are differentially expressed and down-regulated in a developmental stagespecific manner in murine gamma delta T-cells.

The RNA expression value of each differentially expressed snoRNA transcript, in wild-type and CD3H deficient mice ( $n=2$ for developmental stage; each $n$ of 2 includes 1 sample from day 18 embryos and adult mice 6 weeks of age) is displayed. The error bars represent the standard deviation of the mean, and the $p$-value is denoted on each graph. Though the $p$-value may be greater than .05 in some cases, these p-values are comparing the significance of the difference between the actual RNA expression values, and not the statistical significance of global differential expression as determined by GEO2R, shown in Table 1. 

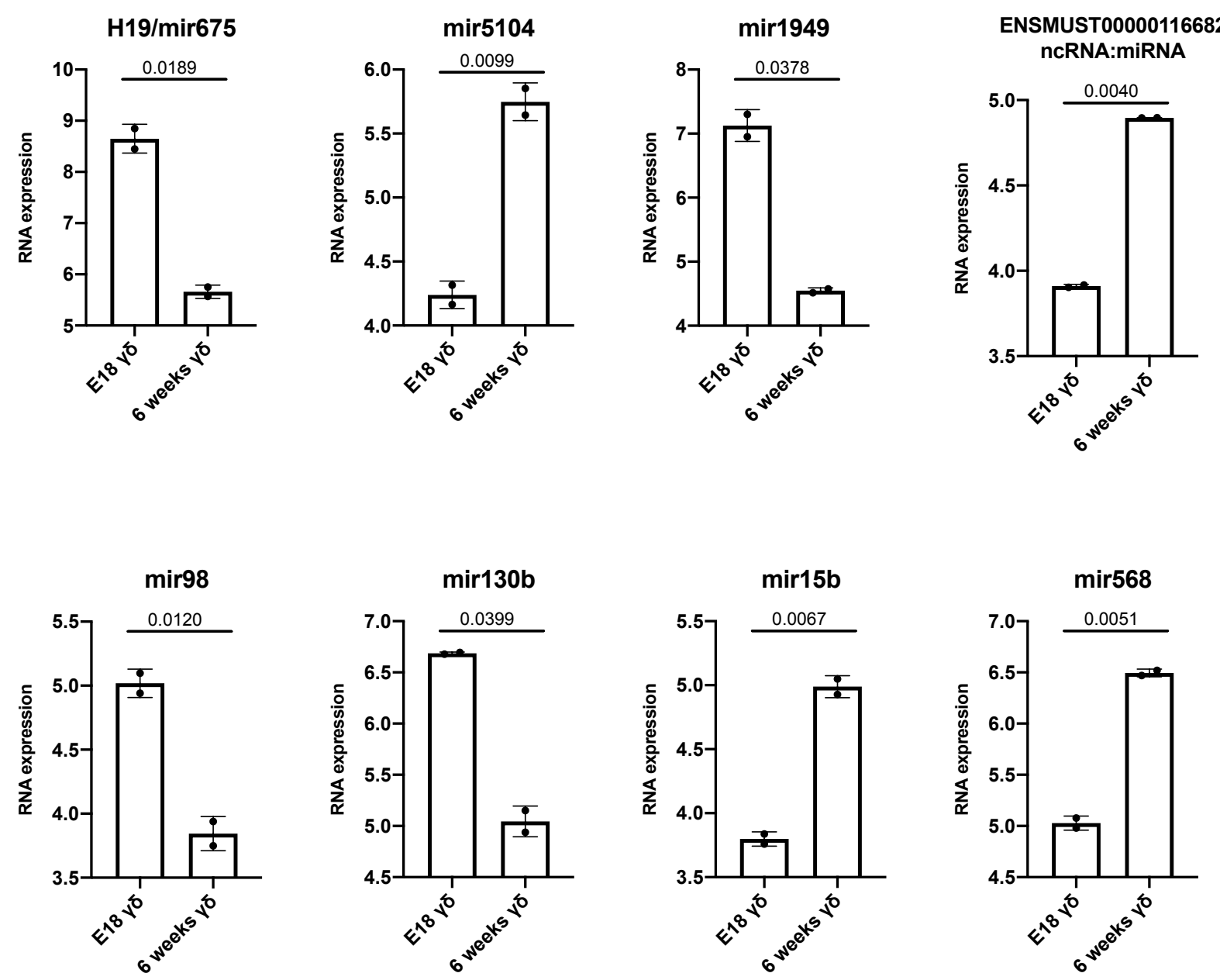

Figure 3: miRNA are differentially expressed in a developmental stage-specific manner in murine gamma delta T-cells.

The RNA expression value of each differentially expressed miRNA transcript, in wild-type and CD3H deficient mice ( $n=2$ for developmental stage; each $n$ of 2 includes 1 sample from day 18 embryos and adult mice 6 weeks of age) is displayed. The error bars represent the standard deviation of the mean, and the p-value is denoted on each graph. Though the p-value may be greater than .05 in some cases, these p-values are comparing the significance of the difference between the actual RNA expression values, and not the statistical significance of global differential expression as determined by GEO2R, shown in Table 1. 